\title{
Judgment of Temporary over Voltages during Transformer Refurbishment
}

\author{
V V Rajagopal Peesapati \\ Electrical and Electronics \\ Engineering Department \\ National Institute of Technology \\ Jamshedpur, India
}

\author{
Niranjan Kumar \\ Electrical and Electronics \\ Engineering Department \\ National Institute of Technology \\ Jamshedpur, India
}

\author{
Vinod Kumar Yadav \\ School of Electrical, Electronics \\ and Communication Engineering \\ Galgotias University \\ Greater Noida, India
}

\begin{abstract}
In this research work Radial Basis Function (RBF) Neural Network (NN) and Regression models are used to estimate Temporary over Voltages (TOVs) that are occurred during the reenergization of lightly loaded transformer. Assessment of the TOVs are carried out in order to control the high peak voltage up to a safe operating point to ensure the safety of the power system equipments. The proposed approaches are applied to the standard IEEE 30 bus test system to evaluate its efficacy. The statistical approach is applied to examine the robustness of the forecasting results. Results show that the methods conveyed in this research are better and could be helpful for power system restoration planning.
\end{abstract}

\section{Keywords}

Power System Restoration, Temporary Over Voltages, Radial Basis Function Neural Networks.

\section{INTRODUCTION}

The power system restoration after a major or a partial blackout is complex and time consuming in nature. This needs several actions to be taken place in very short duration and with more accuracy. The restoration plan consists of several objectives like total system risk, total served energy in the period of restoration and total restoration time [1]. The simulation work helps in the best way of loading the power plants and is applied for hydro power plant and steam power plant restoration [2]. The power system restoration problem involves so many factors like frequency monitoring, black start unit energization, transmission line energization and transformer energization [3]-[5]. Data Envelopment Analysis technique is used to evaluate efficiency of different types of Decision Making Units (DMUs) including transmission lines energization, performance evolutions of different electrical distribution utilities [6]-[8]. A study of TOVs in Vietnam $500 \mathrm{kV}$ system are presented and proposed that installation of Surge Arrester will be helpful for ensuring the safety of major power system equipments [9]. The nonlinearities in transformer core, saturation of transformer are the main causes of harmonics in inrush currents [10]-[12]. The application of Power System Blockset (PSB) in MATLAB/Simulink based simulation tool is discussed and various examples have been analyzed [13]. The Artificial Neural Networks are used as best forecasting techniques in engineering application; these are used in power system restoration problem and applied on 162 bus transmission for analysis [14]. The estimation of temporary over voltages which are occurred during the energization of transformer in the process of restoration is beneficial in designing the minimum rating of devices to be used. The temporary over voltages are reduced by controlling switching, the evaluations of inrush currents for single phase transformer are done on the basis of automatic procedure [15], [16].
The present research work uses the MATLAB/Simulink based simulation tool for analyzing the peak and duration of TOVs for portions of IEEE 30 bus system. The peak and duration of TOVs are estimated through the application of RBFNNs. The optimization is carried out in order to limit the TOVs up to a safe operation point. Further the structure of the paper was as follows after a brief introduction in section 1 , section 2 discusses proposed methodologies in achieving the objectives. The results are analyzed in section 3 and conclusions from this research work are figured out in section 4 .

\section{METHODOLOGIES}

\subsection{Estimation of Temporary over Voltages}

During the restoration process the major concern is related to temporary over voltages caused by transformer energization at lightly loaded condition. The over voltages occurring in the restoration process are estimated through MATLAB/Simulink based simulation tool and the RBFNNs.

\subsubsection{Simulation Networks}

The portion of IEEE 30 bus test system is considered for analysis and implementation purpose in the research work. The test system consists of 41 network lines, 2 generators, 4 synchronous condensers and 2 shunt capacitors. The bus voltage varies from 1.00 per unit (p.u) to 1.082 per unit (p.u) and the phase angles of each bus are considered as zero degrees. The present research work considers $100 \mathrm{MVA}$ as base MVA and $132 \mathrm{kV}$ as base $\mathrm{kV}$. The networks used for the case studies are designed in the MATLAB/ Simulink platform. The generators are designed as voltage sources in series with impedances. The network lines are modeled as PI section lines for every $25 \mathrm{~km}$. The network lines constitute of lumped resistances and inductances. The capacitances are considered at starting and at the end position of the network line section. The power transformers are designed with leakage inductances, copper loss component, core loss components and magnetizing components. The power transformer rated in this research work is considered as 500MVA with a voltage ratio of $132 \mathrm{kV} / 33 \mathrm{kV}$. The core elements of transformer represent the core loss component and magnetization component and the saturation characteristics are specified as a piece wise linear characteristics. The loads constitute of constant impedances $[13,17]$. Here for the analysis two loads connected to two different transformers of the network are considered. The single line diagrams of each case study are shown in fig. $1 \& 2$. 


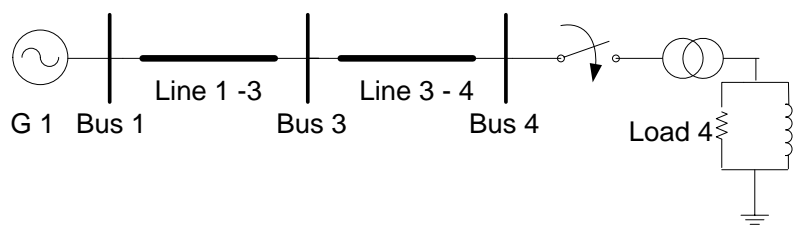

Figure 1. Single line diagram of power system for case 1



Figure 2. Single line diagram of power system for case 2

\subsubsection{Artificial Neural Networks}

Artificial Neural Networks are best in approximation and can be used in many fields like estimation, pattern recognition and control systems. The ANNs are classified into many categories, the most popular category being multi layer perceptron. The model proposed in this research work will use Radial Basis Function Neural Networks, simpler than perceptron [18]. This model is used for the estimation of TOVs during the energization of transformer in the restoration process. Of a set of inputs and a set of outputs the approximation by RBFN will be the weighted sum of $m$ Gaussian kernels $\phi$ as given by the equation below:

$$
\begin{aligned}
& \hat{y}_{t}=\sum_{i=1}^{m} \lambda_{i} \phi\left(x_{t}, P_{i}, z_{i}\right) \\
& t=1 \text { to } N \\
& \text { with } \\
& \phi\left(x_{t}, P_{i}, z_{i}\right)=e^{-\left(\left\|\frac{x_{t}-P_{i}}{\sqrt{2} z_{i}}\right\|\right)^{2}} \\
& \text { where } \\
& \lambda_{i}=\text { the multiplicative factors } \\
& P_{i} \quad=\quad \text { position of Gaussian kernels } \\
& z_{i}=\text { Gaussian kernels variances }
\end{aligned}
$$

The Gaussian kernel position is dependent on the distribution of $x_{t}$ in space. The placement of nodes depends on the number of input data. Vector quantization is the technique that allows the realization of the above operation. The node positions are called centroids. The vector quantization technique comprises two stages, the first being random initialization in the space. All $x_{t}$ points are inspected, and for each of them the closest centroid will be moved in the direction of $x_{t}$ according to the following formula:

$$
P_{i}=P_{i}+\alpha\left(x_{t}-P_{i}\right)
$$

With $x_{t}$ the considered point, $P_{i}$ the closest centroid to $x_{t}$, and a $\alpha$ parameter that decreases with time. Large numbers of testing data have been used to check the proposed solution in the most objective way at practically all possible parameter variations.

\subsubsection{Static Measure to Determine the Accuracy of Forecasts}

To evaluate the forecast performance, the popular Mean Absolute Percentage Error (MAPE) has been used. As error observation in the MCP characteristics are important for the forecasting process. The mathematical finding the MAPE is expressed as below.

$$
\% M A P E=\frac{1}{N} \sum_{i=1}^{N} \frac{\mid \text { Actual }- \text { forecatsed } \mid}{\text { Actual }} \times 100
$$

\subsection{Optimization of TOVs}

MATLAB Optimization tool box has many inbuilt functions like fmincon, fminimax, fminsearch, fsolve, fzero and many more. The function fminicon is popular as it finds a minimum of scalar function of several variables starting at an initial estimate. This can also be referred to as constrained nonlinear optimization or nonlinear programming. The objective function of fminicon function is defined as below

$$
\min f(x)
$$

The objective function above stated is minimized bounded to several constraints. The various constraints are denoted as follows:

Linear inequalities, these are the inequality constraints of the variable $X$ and can be determined by equation () as shown below. If no inequalities exist the constraints matrices $\mathrm{A} \& \mathrm{~b}$ are passed with empty matrices respectively.

$$
A * x \leq b
$$

Linear equalities, these are the linear equality constraints of the variable $x$ and are as shown below

$$
A_{e q} * x=b_{e q}
$$

If no equalities exist then $A_{e q}=[]$ and $b_{e q}=[]$

Lower \& Upper Bounds, these are the lower and upper limits within which the variable lies. If the variable is unbounded below then the lower bound value will be $-\infty$ and unbounded above then the upper bound value will be $\infty$.

$$
\text { If there are no bounds exist the } l b=[] \text { and } u b=[]
$$

The present research work minimizes the voltage function of TOVs using the MATLAB optimization tool box.

\section{RESULTS AND ANALYSIS}

The present work estimates the peak and duration of TOVs that have occurred during the process of restoration by both Simulink and Artificial Neural Networks. The artificial Neural Networks are trained with different obtained from simulation tool. The ANNs are tested with the data which was unseen by it ever. The MATLAB/ Simulation tool [13] is applied to a section of IEEE 30 bus test system to measure the values of the voltage peaks and time duration of the peak voltage ( $>1.4$ p.u) at the transformer buses as shown in both single line diagrams of fig $1 \& 2$. 


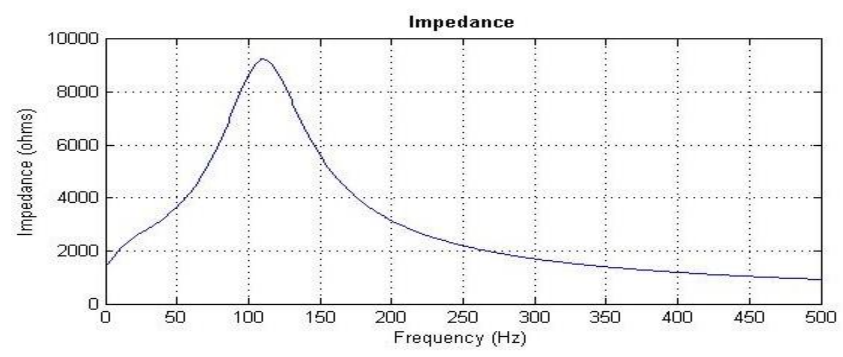

Figure 3 Shows Impedance versus frequency response of network 1

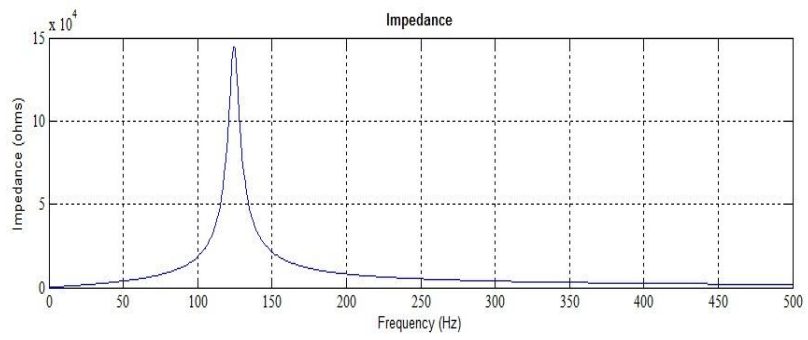

Figure 4 Shows Impedance versus frequency response of network 2.

The generators in both the single line diagrams are non black start units. The serving of the load is done through the energization of transformers at buses $4 \& 6$ respectively in further restoration steps. During this process of restoration the transformer was energized and TOVs are occurred due to light loaded condition of the transformer. The parallel resonance peak occurred at $110 \mathrm{~Hz}$ and $125 \mathrm{~Hz}$ for the networks $1 \& 2$ as shown in figures $3 \& 4$ respectively. The maximum peak in the network-1 and network- 2 is observed as $2.28 \mathrm{p}$. u. and $1.78 \mathrm{p}$. u. respectively for the source voltage of 1.125 p.u. The peaks $(>1.4$ p.u) resided for an average of 0.003703 seconds in the first network and for an average of 0.001440 seconds in the second network. The ANNs were trained with the goal of error minimum and the estimated values of the peaks. The ANN results show a complete agreement with the MATLAB/ Simulink values. The MAPEs presented in the table 2 are very low as compared to other conventional models. The results show that the method used for estimation in this research work is more sensitive and accurate in forecasting the output when compared with the other conventional models. The estimation of TOVs are needful in designing the withstand voltage of the devices used at the instant of reenergization of network lines.

Table 2. \% MAPEs obtained through ANN results

\begin{tabular}{c|c|c}
\hline Circuit & $\begin{array}{c}\text { \% MAPE } \\
\text { (Voltage) }\end{array}$ & $\begin{array}{c}\text { \% MAPE } \\
\text { (Duration) }\end{array}$ \\
\hline $\begin{array}{c}\text { Single line } \\
\text { diagram - }\end{array}$ & 0.72608 & 0.71251 \\
\hline $\begin{array}{c}\text { Single line } \\
\text { diagram }-2\end{array}$ & 1.256567 & 2.187538 \\
\hline
\end{tabular}

With the help of MATLAB/ Simulink the TOV that occurred during the energization of lightly loaded transformer are optimized. The optimized results for the both networks are shown in table 3.
Table 3. Results of Optimization for the both networks

\begin{tabular}{c|c|c}
\hline $\begin{array}{c}\text { Source voltage } \\
\text { (p.u.) }\end{array}$ & $\begin{array}{c}\text { Optimized voltage for } \\
\text { Network }-1 \\
\text { (p.u.) }\end{array}$ & $\begin{array}{c}\text { Optimized voltage for } \\
\text { Network }-2 \\
\text { (p.u.) }\end{array}$ \\
\hline 0.925 & 1.5213 & 1.3228 \\
\hline 0.975 & 1.5316 & 1.3342 \\
\hline 1.025 & 1.5432 & 1.3456 \\
\hline 1.075 & 1.6588 & 1.3476 \\
\hline 1.100 & 1.6917 & 1.3566 \\
\hline 1.125 & 1.7172 & 1.6244 \\
\hline
\end{tabular}

\section{CONCLUSIONS}

This research work presents an alternate estimation technique for forecasting the peak and duration of TOVs that are occurred during the reenergization of lightly loaded transformer. The estimation results reveal that the TOVs in magnitude are very high and need to be controlled in order to ensure safety for the power system equipments. The proposed method estimates the TOVs with a good accuracy as compared to conventional methods. In addition to the estimation the TOVs are optimized to determine safe operating points for different source voltages. The proposed research work will be helpful at early stages for power system restoration planning after occurring a complete or a partial blackout situation. The optimized values of Temporary Over Voltages are useful in determining the device withstand voltages to be used at the instant of reenergization.

\section{REFERENCES}

[1] Saber Nouri-Zadeh and Ali Mohammad Ranjbar, "Multi objective power system restoration," in Proc.2008 IEEE Power and Energy Conf., pp. 1-5.

[2] V. Yari, S. Nourizadeh and A. M. Ranjbar, "Determining the Best Sequence of Load Pickup During Power System Restoration," in Proc. $20109^{\text {th }}$ Int. Conf. Environmental and Electrical Engineering, pp. 1-4.

[3] Saber Nourizadeh, Vahid Yari and Ali Mohammad Ranjbar, "Frequency Monitoring and Control during Power System Restoration Based on Wide Area Measurement System," Mathematical Problems in Engineering, vol. 2011. pp. 1-13, Jan. 2011.

[4] Jhon H. Brunke and Klaus J. Frohlich, "Elimination of Transformer Inrush Currents by Controlled Switching-Part II: Application and Performance Considerations," IEEE Trans. Power Delivery, vol. 16. pp. 281-285. Apr. 2001.

[5] En Lu, Zhijun Qin, Mingbo Liu, Yunhe Hou, Ning Wang and Jinyu Wen, "Increasing Black Start Capacity by Fast Cut Back Function of Thermal Power Plants," Int. Jour Smart Grid and Clean Energy, vol. 2. pp. 60-66. Jan. 2013.

[6] LiBao Shi, H. L. Ding, and Zaho Xu, "Determination of Weight Coefficient for Power System Restoration," IEEE Trans. Power Syst., vol. 27, no. 2, pp. 1140-1141, May 2012.

[7] Yadav VK, Padhy NP and Gupta HO, "Performance evaluation and improvement directions for an Indian electric utility," Energy Policy, vol. 39, pp. 193-205, 2011.

[8] Vinod Kumar Yadav, Yogesh K. Chauhan, N. P. Padhy and H. O. Gupta, "A novel power sector restructuring model 
based on Data Envelopment Analysis (DEA)," Elect. Power and Energy Syst. vol 44, pp. 629-637, 2013.

[9] S. Lam-Du, T. Tran-Quoc, N. Nguyen-Khac, R. Feuillet, J. C. Sabonnadiere, K. Nguyen-Boi, Q. Nguyen, "Temporary Over Voltages in The Vietnam 500kV Network," in Proc. 1998 Int. Conf. Energy Management and Power Delivery. pp. 275-280.

[10] C. E. Lin, C. L. Cheng, C. L. Huang, and J. C. Yeh, "Investigation of magnetizing inrush current in transformers Part II - Harmonic Analysis," IEEE Trans. Power Delivery, vol. 8, pp. 255-263, Jan. 1993.

[11] N. Rajakovic, and A. Semlyen, "Investigation of the inrush phenomenon a quasi-stationary approach in the harmonic domain," IEEE Trans. Power Delivery, vol. 4, pp. 2114 2120 , Oct. 1989.

[12] J. F. Fuller, E. F. Fuchs, and K. J. Roesler, "Influence of harmonics on power distribution system protection," IEEE Trans. Power Delivery, vol. 3, pp. 549-557, Apr. 1988

[13] G. Sybille, P. Brunelle, L. Hoang, L. A. Dessaint, and K. AlHaddad, "Theory and applications of power system blockset, a MATLAB/Simulink-based simulation tool for power systems," in Proc.2000 IEEE Power Eng. Soc. Winter Meeting, pp. 774-779.

[14] Arturo S. Bretas and Arun G. Phadke, "Artificial Neural Networks in Power System Restoration," IEEE Trans. Power Delivery, vol. 18. pp. 1181-1186, Oct. 2003.

[15] Abbas Ketabi, Ali Mohammad Ranjbar and René Feuillet, "Analysis and Control of Temporary Overvoltages for Automated Restoration Planning," IEEE Trans. Power Delivery, vol. 17. pp. 1121-1127. Oct2002.

[16] Iman Sadeghkhani, Abbas Ketabi and Rene Feuillet, "New Approach to Harmonic Overvoltages Reduction during Transformer Energization via Controlled Switching," in Proc. $200915^{\text {th }}$ Int. Conf. Intelligent System Applications to Power Systems. pp.1-6.

[17] Emil Cazacu, Valentin Ionita and Lucian Petrescu, "An Improved Method for the Inrush Current Evaluation in Single Phase Power Transformers," in Proc. 2013 8th Int. Symposium on Advanced Topics in Electrical Engineering, pp. 1-6.

[18] S. A. Kalogirou, "Artificial neural networks in renewable energy systems applications: a review". Renewable and Sustainable Energy Reviews, vol.5, pp.373-401, 2000. 